\title{
B lymphocyte accumulations in human pulmonary sarcoidosis
}

\author{
S B Fazel, S E M Howie, A S Krajewski, D Lamb
}

\begin{abstract}
Background Although cell mediated immunity is primarily thought to mediate the pathogenesis of sarcoidosis, the presence of immunogtobutins, immune complexes and complement suggests that processes of humoral immunity may contribute to immunopathology in sarcoid lesions. To test this hypothesis, the distribution of $B$ lymphocytes in paraffin embedded sarcoid lesions in mediastinal lymph nodes and open lung biopsy specimens was investigated.
\end{abstract}

Methods Paraffin sections from eight open lung and 21 lymph node biopsies from sarcoid patients and five normal and five tuberculous lymph nodes from patients with tuberculosis were stained with a panel of monoclonal antibodies by means of avidin/biotin enhanced immunocytochemistry.

Results Immunohistochemical analysis of the 29 biopsy specimens from the sarcoid patients revealed large numbers of $B$ cells in the intergranulomatous regions. Further investigations in the open lung biopsy specimens indicated that these $B$ cells were often organised into discrete circular or oval shaped aggregates with no germinal centre morphology, in which a few CD45RO memory $T$ lymphocytes were scattered. The $B$ cells were polyclonal, and a few plasma cells (IgM +, IgA +, IgG +) were identified.

Conclusions The finding of large numbers of $B$ lymphocytes in sarcoid pulmonary lesions is in contrast to bronchoalveolar lavage studies, which have demonstrated proportions of $5 \%$ or less of $B$ cells as a total of all immune cells, and therefore indicates that bronchoalveolar lavage may not correctly sample the immune cells of lung interstitial tissue in pulmonary sarcoidosis. The $B$ cells at these sites are the possible origin of some of the humoral changes in the serum and lesions of sarcoid patients. They may also influence the pathogenesis of the disorder by presenting antigen(s) and forming immune complexes at sites of disease activity.

\section{(Thorax 1992;47:964-967)}

Pulmonary sarcoidosis is a chronic granulomatous disease which is thought to be characterised by a mononuclear cell infiltrate in the pulmonary interstitium that precedes the development of non-caseating granulomas. ${ }^{1}$ Although the aetiology of sardoidosis remains unknown, a considerable amount of new information on pathogenesis has been acquired by immunohistological analysis of frozen tissue biopsy specimens and bronchoalveolar lavage fluid (BAL). Together these have given a picture of pulmonary sarcoidosis as a local cell mediated immune response induced by unknown antigen(s), resulting in the activation of $\mathrm{CD} 4+\mathrm{T}$ lymphocytes in an environment lacking adequate numbers of regulatory $\mathrm{CD} 8+$ $T$ cells. These circumstances initially lead to an alveolitis and subsequently to the formation of granulomas as lymphokines released by the activated lymphocytes react with the macrophage populations. ${ }^{1-3}$

Besides changes in $T$ cell and macrophage populations, changes in the humoral immunity have been reported. Polyclonal hypergammaglobulinaemia with increased titres of serum antibodies against a number of antigens and self antigens is found. ${ }^{45}$ The presence of immunoglobulins, immune complexes and complement in sarcoid granulomas and BAL suggests that humoral mechanisms may be involved in the pathogenesis of sarcoidosis..$^{6-7}$ However, previous work on frozen tissue sections and analysis of BAL have not reported significant numbers of $B$ lymphocytes at sites of disease activity. ${ }^{8-12}$ To examine the distribution of $B$ and $T$ lymphocytes in more detail in sarcoid lesions, paraffin reactive cell markers were used on archival mediastinal lymph node and open lung biopsy sections.

\section{Materials and methods}

BIOPSY MATERIAL

The study was carried out on archival material from 29 patients who were clinically and radiologically suspected to have sarcoidosis, and in whom the diagnosis had been confirmed by histopathological demonstration of noncaseating epithelioid cell granulomas in biopsy material obtained from lung or mediastinal lymph nodes. The patients had received no therapy before biopsy. No patients showed signs of concomitant infection or autoimmune disease or had rheumtoid arthritis. The tissue samples were fixed in $4 \%$ buffered formaldehyde and embedded in paraffin wax, and stored in the department of pathology. Of the 29 cases of sarcoidosis investigated, eight had open lung biopsies and 21 lymph node biopsies. Five normal and five tuberculous lymph nodes from tuberculosis patients were used as controls. 
IMMUNOHISTOCHEMICAL STUDIES

Sections were stained by the avidin/biotin complex method. The first protocol was a single step immunoperoxidase method. The substrate reaction was then visualised by application of excess 3,3-diaminobenzidine tetrachloride (appearing brown). A double staining method was also used, with two successive indirect immunoenzyme techniques using different substrate reactions. The first antibody was visualised as above; the monoclonal antibody of different specificity was visualised by rabbit/ anti-mouse immunoglobulin and avidin/biotin complex (with biotinylated alkaline phosphatase solution), followed by alkaline phosphatase staining with Fast Blue BB. (All econdary antibodies and $\mathrm{ABC}$ complexes were obtained from Dako UK Ltd.) After immunostaining, sections were counterstained in Harris' haematoxylin and Scott's tap water solution.

The following paraffin tissue reactive antibodies were used: anti CD45 (leucocyte common antigen), anti-CD20 (L26, B lymphocyte specific), MAC387 (anti unclustered monocyte/macrophage specific determinant), all from Dako UK Ltd; anti CD45R (MB1, restricted to $B$ lymphocytes and naive $T$ lymphocytes), anti-CD43 (MTI, predominantly found on T lymphocytes and macrophages), both from Europath Ltd, UK; anti-CD45RA (F8-11-13, restricted to B lymphocytes and naive $\mathrm{T}$ lymphocytes), the gift of $\mathrm{Dr} \mathrm{R}$ Dalchau; anti-CD45RO (UCHL1, restricted to memory T lymphocytes), the gift of Professor PCL Beverley; anti-CD23 (BU38, found on activated $B$ lymphocytes and germinal centre dendritic cells) from the Binding Site UK Ltd.

\section{Results}

HISTOLOGICAL FEATURES OF SARCOID MATERIAL In sections stained routinely with haematoxylin and eosin, mediastinal lymph nodes and areas of sarcoid lung showed replacement of normal structures by numerous discrete, noncaseating epitheloid cell granulomas, within which were small numbers of lymphocytes and multinucleate giant cells, mostly of the Langhan's type. These granulomas showed no signs

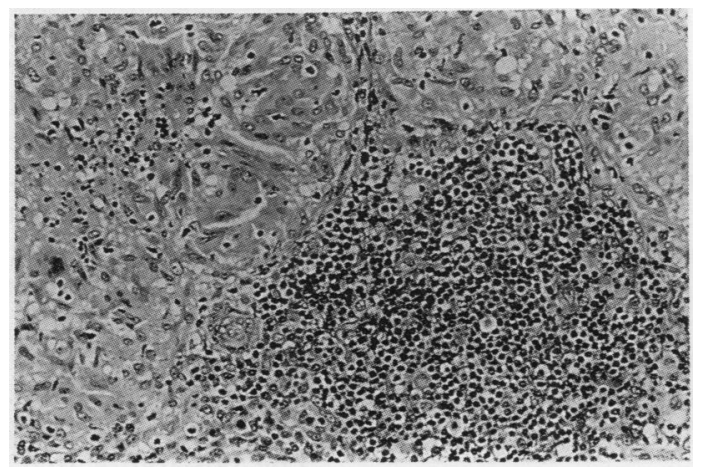

Figure 1 Sarcoid lymph node stained with L26 antiCD20 B lymphocyte specific monoclonal antibody showing the typical appearance of sarcoid nodes. The $B$ cell aggregate lacks the stuctured appearance of a germinal centre. Most of the cells are small lymphocytes with a few plasma cells.

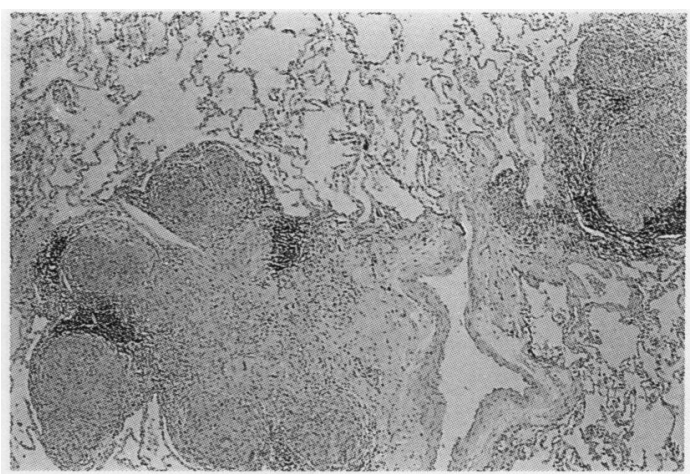

Figure 2 Sarcoid lung stained with L26 anti-CD20 B lymphocyte specific monoclonal antibody. B lymphocyte aggregates can be seen between granulomas, some being adjacent to air spaces.

of necrosis. Regions of variable cellularity were seen between the granulomas, consisting predominantly of lymphocytes with a few scattered plasma cells.

\section{IMMUNOHISTOCHEMISTRY}

Lymph nodes

Normal lymph nodes showed that CD45RO+, CD43 + memory $\mathrm{T}$ lymphocytes were largely confined to the paracortex, where CD45RA +, $\mathrm{CD} 43$ + naive $\mathrm{T}$ cells were also found. The germinal centre lymphocytes were predominantly CD20+, CD45RA + B cells with a few memory CD45 RO + T cells scattered between them. Anti-CD23 stained dendritic reticular cells within germinal centres of follicles and gave weak staining of mantle zone lymphocytes.

Sections of sarcoid nodes demonstrated typical non-caseating granulomas with large numbers of B lymphocytes (CD20+, CD45RA +, CD23 weakly positive) distributed in the intergranulomatous areas (fig 1). Most of the other lymphocytes stained with UCHL1, and the epithelioid cells stained with MT1. These B lymphocyte aggregates consisted predominantly of small B lymphocytes with a few plasma cells. A small number of dendritic reticular cells $(\mathrm{CD} 23+)$ were seen at the margin of a few of the B cell aggregates in each case. However, germinal centres were not seen and no centroblasts or centrocytes were identifiable - that is, these aggregates almost certainly do not represent residual $\mathrm{B}$ cell follicles within the lymph nodes.

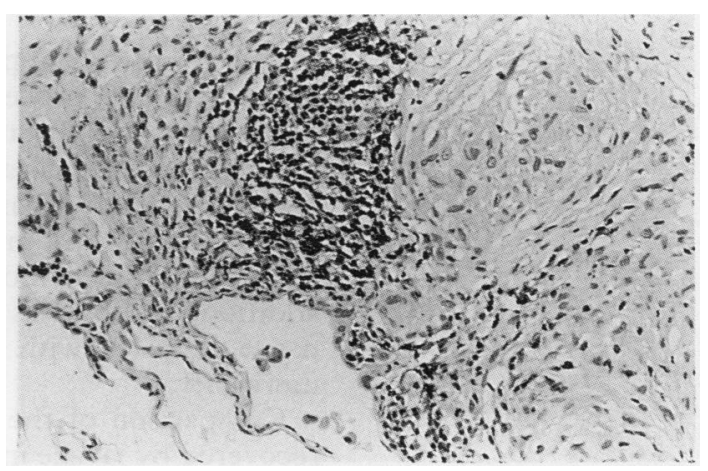

Figure 3 Higher magnification of figure 2 showing that the appearance of lung $B$ cell aggregates is very similar to those in the lymph nodes ( fig 1 ). 


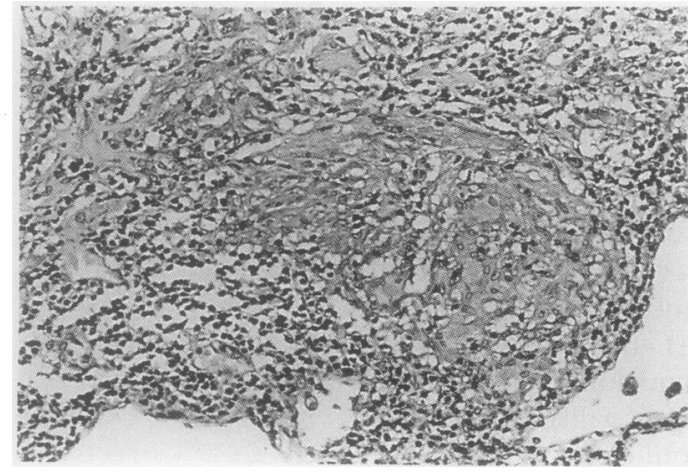

Figure 4 Sarcoid lung stained with UCHL1 antiCD45RO monoclonal antibody showing memory $T$ lymphocytes. Original magnification $\times 200$.

The tuberculous lymph nodes were affected to a variable extent by small and large confluent epithelioid granulomas. The smaller granulomas consisted of collections of epithelioid cells (CD43 positive) and multinucleate giant cells. The large granulomas showed varying amounts of caseation necrosis. Most of the granulomas were surrounded by memory $\mathrm{T}$ lymphocytes (CD45RO+, CD43+), and a number of B lymphocyte aggregates (CD20+, CD45RA + ) were identified.

\section{Sarcoid lung lesions}

In all cases aggregates of B cells $(\mathrm{CD} 20+$, CD45RA +, CD45R +) were found in the intergranulomatous regions (figs 2 and 3 ). Germinal centres containing centroblasts and centrocytes were absent in these B cell aggregates, although follicular dendritic cells staining with anti-CD23 were occasionally present in small numbers. A few scattered $\mathrm{CD} 45 \mathrm{RO}+\mathrm{T}$ lymphocytes were found within the $B$ cell aggregates. All the lymphocytes within granulomas were of the memory cell phenotype (CD45RO + CD43+; (fig 4). The epithelioid cells of the granulomas stained with anti-CD43. Further imunohistochemical studies demonstrated that the B lymphocyte population was polyclonal. A small number of plasma cells was present. These were shown to be mostly IgM + and IgA + with a smaller number of IgG + plasma cells. No IgD+ plasma cells were found. Both kappa and lambda chains were present.

\section{Discussion}

The experimental approach of choice to investigate immune processes in pulmonary sarcoidosis has been bronchoalveolar lavage. This method, because it can be used easily, safely, and repetitively, has become the primary means of assessing inflammation of the lower respiratory tract in most interstitial diseases. In addition, it obviates the need for repeated tissue biopsies with their attendant risk or morbidity. ${ }^{13}$

Comparison of the effector cell population recovered by lavage in sarcoid patients, as well as in other interstitial lung diseases, with transbronchial biopsy in the same subjects, has been reported to have shown that lavage accurately reflects the types and state of activation of the effector cells present in the lungs. Recent comparisons of lavage and frozen tissue lung histology in patients with sardoidosis have concluded that lavage correctly samples not only the cells present at the epithelial surface of alveolar structures, but also inflammatory and immune effector cells throughout the lung paranchyma, ${ }^{8-12}$ although epithelioid cells are rarely seen in the lavage fluid of patients with active sarcoidosis. Lymphocytes recovered in lavage fluid are predominantly $T$ cells and, of several studies since 1984 that have quantified $B$ lymphocyte numbers, none has reported more than a $5 \%$ proportion of $B$ cells. ${ }^{8-12}$ Of the many immunohistological studies conducted on human sarcoid frozen tissue, ${ }^{914-17}$ none has reported the finding of $B$ cell aggregates in sarcoid lesions. Most had not specifically stained for B cells, although one paper describes the presence of large numbers of $B$ lymphocytes forming a "mantle" around the granuloma. ${ }^{16}$

In contrast, the present study of formalin fixed rather than frozen biopsy material demonstrates the presence of large aggregates of $B$ lymphocytes in the lung interstitium in the same areas as the $T$ cells.

Immunohistological analysis does not provide an obvious explanation for this difference between the proportions of B lymphocytes found in tissue sections of lesions and in lavage fluid. $B$ and $T$ cells are found in similar areas of the interstitium. Part of the reason may lie with the fact that in active sarcoidosis, because the number of $T$ lymphocytes is increased, the proportion of B lymphocytes may be decreased. However, as the total number of lung inflammatory and immune effector cells is greatly increased, the absolute number of $B$ cells in the alveolar structure is also probably increased. Another reason may relate to the physical properties of the B cells. It is of interest that $B$ and $T$ cells can be physically separated from one another in vitro by passing mixed suspensions over nylon wool: $T$ cells pass through the wool leaving the "stickier" $B$ cells behind. ${ }^{19} \mathrm{~A}$ similar phenomenon may be occurring in lavage fluid.

The accumulation of $B$ cells during the disease process may be explained by cytokine release by local $\mathrm{T}$ cells. The $\mathrm{T}$ lymphocytes extracted from sarcoid pulmonary lesions have been shown in vitro to secrete significant amounts of B cell differentiation factors IL-2 and IL-4. ${ }^{1}$ It is therefore proposed that the B cells are polyclonally activated to proliferate and differentiate in situ by the activated $T$ cells in the lesions. This is supported by the observations in this study that the B cells were polyclonal with respect to immunoglobulin type, that plasma cells were present in increased numbers, and that $T$ cells were distributed within and proximal to the $B$ cells. The possibility that the $B$ cells, although of different clonal origin, might be recognising related epitopes on an unknown antigenic moiety should not be excluded. This would lead to formation of immune complexes, which have been detected in the serum of patients 
with sarcoidosis. ${ }^{7}$ This process of B cell aggregate (not germinal centre) formation is not specific to sarcoid as similar findings were made in tuberculous tissue, and may rather reflect common pathways of immune system activation and associated damage consequent upon granuloma formation. The $B$ cell aggregates once formed may contribute to the chronicity of the condition.

According to current concepts, the processes of cell mediated immunity are thought to mediate the pathogenesis of sarcoidosis. The changes in humoral immunity are thought to be secondary, and by implication unimportant. In contrast we would like to propose that the accumulation of $B$ cell aggregates at sites of granuloma formation is contributing to the potentiation of the inflammatory process in the tissues and that, although probably occurring after granuloma formation, is not easily dismissable as unimportant in chronic pulmonary sarcoidosis.

A growing body of work from a number of laboratories, including studies by Kurt-Jones et $a l^{14}$ and Ron and Sprent, ${ }^{20}$ suggest that the B cells have an antigen presenting role in vivo. This raises the possibility that the $B$ cells in sarcoid lungs may be presenting antigen and contributing to the persisting immunological reaction at such sites. Further studies investigating the function of $B$ cells in sarcoid lesions will be necessary to clarify their precise role.

We would like to thank Elizabeth Ramage, John Lauder and David Cossar for technical assistance. This work was supported by a British Lung Foundation Grant to SEMH.

1 Thomas PD, Huninghake GW. Current concepts of the pathogenesis of sarcoidosis. Am Rev Respir Dis 1987;135:747-60.

2 Crystal RG, Roberts WC, Hunninghake GW, Gadek JE, Fulmer JD, Line BR. Pulmonary sarcoidosis: a disease characterized and perpetuated by activated lung $T$ lymphocytes. Am Intern Med 1981;94:73-94.
3 Poulter LW. Immune aspects of sarcoidosis. Postgrad Med J 1988;64:536-43.

4 Hunninghake GW, Crystal RG. Mechanisms of hypergam maglobulinaemia in pulmonary sarcoidosis. $J$ Clin Invest $1981 ; 67: 86-92$.

5 Spurzem JR, Saltini C, Crystal RG. Functional significance of anti-T lymphocyte antibodies in sarcoidosis. $A m R e v$ Respir Dis 1988;137:600-5.

6 Dall'Aglio PP, Pesci A, Bertorelli G, Brianti E, Scarpa S. Study of immune complexes in bronchoalveolar lavage fluids. Respiration 1988;54(Suppl 1):36-41.

7 Daniele RP, McMillan LJ, Dauber JH, Rossman MD Immune complexes in sarcoidosis. Chest 1978;74:261-4.

8 Ainslie GM, Poulter LW, Du Bois RM. Relation between immunocytological features of bronchoalveolar lavage fluid and clinical indices in sarcoidosis. Thorax 1989;44:501-9.

9 Campbell DA, Poulter LW, Du Bois RM. Immunocompetent cells in bronchoalveolar lavage reflect the cell populations in transbronchial biopsies in pulmonary sarcoidosis. Am Rev Respir Dis 1985;132:1300-6.

10 Ceuppens JL, Lacquet LM, Marien G, Demedts M, Van der Eeckhout A, Stephens E. Alveolar T cell subsets in pulmonary sarcoidosis. Am Rev Respir Dis 1984;129. 563-8.

11 Hance AJ, Saltini C, Crystal RG. Does de novo immunoglobulin synthesis occur in the epithelial surface of the human lower respiratory tract? Am Rev Respir Dis 1988;137:17-24.

12 Paradis IL, Dauber JH, Rabin BS. Lymphocyte phenotypes in bronchoalveolar lavage and lung tissue in sarcoidosis and pulmonary fibrosis. Am Rev Respir Dis 1986;133:85560.

13 Reynolds HY. Bronchoalveolar lavage and lung histology. Am Rev Respir Dis 1987;135:250-63.

14 Semenzato G, Agostini C, Zambello R, Trentin L, Chitosi $M$, Angi MR et al. Activated T cells with immunoregulatory functions at different sites of involvement in sarcoidosis. Ann NY Acad Sci 1986;465:56-73.

15 Viale G, Codecasa L, Bulgheroni P, Giobbi A, Madonni E, Dell'Orto $P$ et al. T cell subsets in sarcoidosis. Hum Pathol 1986;17:476-81

16 Van den Oord JJ, De Wolf-Peeters C, Facchetti F, Desmet VJ. Cellular composition of hypersensitivity type granulomas. Hum Pathol 1984;15:559-65.

17 Mishra BB, Poulter LW, Janossy G, Geraint James D. The distribution of lymphoid and macrophage like cell subsets of sarcoid and Kveim granulomata. Clin Exp Immunol 1983;54:705-15.

18 Julius MH, Simpson E, Herzenberg LA. A rapid method for the isolaton of functional thymus derived murine lymphocytes. Eur J Immunol 1973;3:645-50.

19 Kurt-Jones EA, Liano AD, Hayglass KA, Benacerraf B, Sy MS, Abbas AK. The role of antigen presenting $B$ cells in $T$ cell priming in vivo: studies of $B$ cell deficient mice. $J$ Immunol 1988;140:3773.

20 Ron Y, Sprent J. T cell priming in vivo: a major role for $B$ cells in presenting antigen to $\mathrm{T}$ cells in lymph nodes. $J$ Immunol 1987;138:2848-56. 